

\title{
Política pública de la migracion en el Departamento del Valle del Cauca ¿Cómo se está tratando el tema de la migración internacional, en el Departamento del Valle del Cauca hasta el 2009?"
}

\author{
Diana Marcela Méndez Figueroa ${ }^{1}$ \\ d.marce65@gmail.com
}

Lorena López López ${ }^{2}$

lorell_21@hotmail.com

\section{Resumen}

El presente documento de trabajo se basa en la tesis de grado, presentada en la Universidad Icesi de Cali, bajo el mismo título del presente artículo. El texto aborda la Política Integral Migratoria (PIM) de Colombia y sus implicaciones en el departamento del Valle del Cauca. El documento está dividido en cuatro temas principales. Primero, se presenta el marco teórico, enfocado, particularmente, en el concepto de migración internacional, el cual se analiza desde distintos puntos de vista y autores.Segundo, se retoman los últimos 30 años de proceso histórico migratorio desde el departamento del Valle del Cauca al exterior.Tercero, se analiza el documento CONPES 3603, emitido por el Gobierno Nacional, en cabeza del Ministerio de Relaciones Exteriores, y se escruta sobre la influencia de este en las políticas formuladas e implementadas en el departamento. Cuarto, se enumeran algunas de las organizaciones gubernamentales, $y$ no gubernamentales, más relevantes con las cuales trabaja el gobierno del Valle del Cauca para lograr el desarrollo de la Política Integral Migratoria (PIM) en el departamento. Finalmente, el presente artículo pretende brindar algunas conclusiones, a modo de reflexión, sobre los retos que enfrenta la Política Integral Migratoria (PIM) en el departamento.

Palabras clave: Migración internacional, Política Integral Migratoria (PIM), trata de personas, remesas, participación civil,inversión extranjera, sistemas de información.

* El presente artículo no representa la posición oficial del Ministerio de Relaciones Exteriores.

1 Diana Marcela Méndez Figueroa, es Contadora Pública y Financista Internacional de la Universidad Icesi. Actualmente trabaja en el Programa Colombia Nos Une del Ministerio de Relaciones Exteriores de la República de Colombia como asesora en la Gobernación del Valle del Cauca, donde coordina, junto con el equipo de Gobierno Departamental, la implementación del Plan de Retorno Positivo.

2 Lorena López López estudiante de Economía y Negocios Internacionales, X semestre de la Universidad Icesi 


\begin{abstract}
The present working paper is an adopted version of the authors' thesis completed in December 2009 at Icesi University in Santiago de Cali- Colombia.The article studies the Colombian Public Policy for Integral Migration (PIM) and its effects in the department of Valle del Cauca. A particular attention is given to the discussion of the relations between the national authority and the departmental government and the impact of a nationally-designed public policy on the society of the region. The document is divided in four topics. First, a conceptual framework devoted to the concept of international migration. Second, a historical of the process of migration in the Department of Valle del Cauca is described for the past 30 years. Third, the paper analyzes the CONPES 3603, document approved by the National Government and designed by the Ministry of Foreign Affairs, in order to estimate its influence on government policies in the Department of Valle del Cauca. Fourth, the paper discusses some of the most relevant organizations working with the government of Valle del Cauca (Colombia) in order to develop a public policy on international migration within the department.
\end{abstract}

Keywords: International Migration, human trafficking, migrant smuggling, remittances, civil participation, foreign investment, information systems.

\section{Introducción}

Las relaciones y la coordinación entre las entidades que trabajan en torno a un mismo tema debe ser la piedra angular para el óptimo desarrollo de las políticas públicas que promuevan el respeto por los Derechos Humanos de la población, especialmente si se trata de migrantes. Por ello, el presente estudio, basado en testimonios y vivencias de organizaciones gubernamentales y no gubernamentales que están trabajando sobre la política pública que busca hacer frente al fenómeno de la migración internacional, pretende dar un acercamiento a la realidad que vive el departamento del Valle del Cauca con relación al fenómeno de la migración internacional.

La situación de la migración internacional, si se tiene en cuenta que el Valle del Cauca presenta el mayor índice de migrantes internacionales de Colombia
(DANE, 2005), es de gran relevancia para el Departamento. Por esa razón, es un tema prioritario de la agenda pública del Gobierno Departamental, que busca encaminar esfuerzos para la creación de políticas públicas que faciliten la protección, el aseguramiento y el cumplimiento de los Derechos Humanos e Internacionales de los connacionales que se encuentran fuera de Colombia, de su país de origen.

De este modo,la Gobernación del Valle del Cauca cedió espacios para que entidades como la Fundación Esperanza, La Comisión Europea de Ayuda al Refugiado (CEAR)y la ONG América, España,Solidaridad y Cooperación (AESCO), desarrollaran un trabajo en torno a las situaciones que se derivan de la Migración Internacional. Estas entidades están ubicadas dentro del Departamento y han promovido numerosas campañas tendientes a resaltar 
las necesidades y problemáticas que se presentan alrededor de los migrantes.

El presente texto está dividido en cuatro temas principales. Primero, se presenta el marco teórico, enfocado, particularmente, en el concepto de migración internacional, analizándolo desde distintos puntos de vista y autores. Segundo, se retoman los últimos 30 años de proceso histórico migratorio desde el Departamento del Valle del Cauca al exterior.Tercero, se analiza el documento CONPES 3603, emitido por el Gobierno Nacional, en cabeza del Ministerio de Relaciones Exteriores, y se escruta sobre la influencia de este en las políticas formuladas e implementadas en el Departamento. Cuarto, se enumeran algunas de las organizaciones gubernamentales, y no gubernamentales, más relevantes con las cuales trabaja el gobierno del Valle del Cauca para lograr el desarrollo de la Política Integral Migratoria (PIM) en el Departamento. Finalmente, el presente artículo pretende brindar algunas conclusiones, a modo de reflexión, sobre los retos que enfrenta la Política Integral Migratoria (PIM) en el Departamento.

\section{i. Enfoque teórico}

El Valle del Cauca es uno de los principales departamentos de Colombia que presenta actualmente fenómenos de migración humana internacional. Según cifras oficiales, el $24,1 \%$ de los tres millones tres mil colombianos residentes fuera del país son vallecaucanos (DANE -Departamento Administrativo Nacional de Estadísticas, 2005).

Con el fin de poder describir las situaciones que explican esta cifra de participación de los vallecaucanos dentro del total de la población migrante en el país, es preciso comprender el concepto de migración y cómo la globalización lo ha moldeado.Lo cual se hace a continuación en este texto.

Existen diferentes fuentes que explican el término "migración" desde distintos enfoques. Desde el punto de vista espacial o geográfico (1), la migración es inversamente proporcional a la distancia; desde sus causas (2), pueden ser voluntarias en casos políticos (guerras y/o persecuciones) o forzadas en lo económico, social o individual; y desde lo demográfico (3), los desplazamientos humanos son el resultado de factores económicos específicos, en particular, la necesidad de trabajos especializados que escasean en un país y abundan en otros. ${ }^{3}$ Por su parte, Carassou propone otro paradigma, basado en la Teoría de la modernización: "las migraciones se inscriben en la fase de la movilización de masas como una respuesta, consecuencia y expresión del proceso de modernización percibido en su conjunto". ${ }^{4}$ A su vez, Germani toma la migración como un proceso de modernización en el que evidencia dos problemas: uno de aspecto macroteórico -el cambio social expresado en la modernización-, y otro a nivel microteórico - el proceso de toma de decisión.

No obstante, la Organización Internacional para las Migraciones (oIM) entiende la migración como

"el movimiento de una persona o grupo de personas de una unidad geográfica a

3 Herrera Carassou, Roberto.La perspectiva teórica en el estudio de las migraciones, 2006, 227 págs.

4 Organizacíon Internacional de Migración (OIM), Migración e Historia, http://www.iom.int/Jahia/ migration-management-foundations/migrationhistory/cache/offonce/leng/es. Revisado septiembre 
otra, a través de fronteras administrativas o políticas, que desean establecerse definitiva o temporalmente, en un lugar distinto a su lugar de origen". ${ }^{5}$

Un punto en común de las referencias antes mencionadas, que corresponde a fronteras, modernización y desplazamientos humanos, sería el término "globalización”, comprendido como un proceso de libre circulación, donde la movilidad de los diversos factores económicos, sociales y políticos se convierte en una necesidad latente para la situación de una economía local. Los mercados mundiales son ahora sinónimo de integración, donde los progresos tecnológicos han mejorado la comunicación a través de diversas herramientas. Precisamente, ese cambio tecnológico ha fomentado el deseo o la necesidad imperativa de traslado a otro país. [Meyer, 2006:90-125pg].

“Globalización” no sólo debe entenderse como un término que atañe a los procesos de internacionalización; debe comprenderse, además, en relación con la importancia que tiene ella en las diferentes instancias del poder político y su efecto en la comunidad.

"Uno dice globalización y se piensa en algo que está por fuera de todas las instituciones. Lo que sucede es que el Estado se globaliza, especialmente el Poder Ejecutivo, pero la Legislatura se domestica”. ${ }^{6}$

Así mismo, Sassen presenta su argumento sobre la Teoría de la Globalización argumentando que "la soberanía se haya visto transformada y que la territorialidad exclusiva, una característica distintiva del estado moderno, esté siendo socavada

5 oIM, www.oim.org

6 Sassen, Saskia. Una sociología de la globalización, Katz Editores, Argentina, 2007. por la globalización económica". ${ }^{7}$ De esta manera encontramos que, dentro de la concepción teórica del proceso, "la globalización está desafiando la estabilidad y la territorialidad del estado, así como su capacidad para controlar sus políticas económicas y del bienestar". ${ }^{8}$

"La migración internacional forma parte esencial de la globalización”. 9 "Por lo tanto, si los gobiernos defienden la libertad de circulación del capital, de bienes y de ideas, es poco probable que sean capaces de frenar la circulación de personas". ${ }^{10}$ Es así como se relaciona la migración internacional con el enfoque transnacional, dado que, como explica Castles, "si la movilidad transnacional es parte de la vida económica, social, cultural y política de un grupo, esto brinda una motivación poderosa para superar las barreras impuestas por los estados". ${ }^{11}$

El término "transnacional" implica aquellas actividades humanas e instituciones sociales que se extienden a través de las fronteras nacionales. La propia definición de transnacionalismo se refiere tanto a los estados como a sus fronteras, que son cruzadas por flujos de gente, dinero o conformación y se expanden por medio de redes, organizaciones o campos [Bauböck, 2003:2].

7 Sassen, Saskia. Beyond Sovereignty: Inmigration policie Making today, Social Justice, 23(3):9-20,1996a. 8 Meyer, Eytan. Teoría de las políticas migratorias, un análisis comparado, Zona Abierta, España, 2006, 90-125pg.

9 Castles S. Globalization and migration: Some Pressing Contradictions, International Social Science Journal,1998:182.

10 Meyer, Eytan. Teoría de las políticas migratorias, un análisis comparado, Zona Abierta, España, 2006, 90-125pg.

11 Castles, Stephen, Factores que hacen y deshacen las políticas migratorias, 2006. 
Alejandro Portes define al transnacionalismo como un concepto que "se relaciona cada vez más con los campos sociales que crean los inmigrantes entre sus comunidades y naciones de origen, generalmente pobres, y los países avanzados donde se establecen". ${ }^{2}$

Según las conclusiones del Seminario Internacional sobre migración internacional colombiana y la conformación de comunidades transnacionales, "el Transnacionalismo son las relaciones continuas entre los migrantes y sus países de origen y cómo esas relaciones de doble vía, van construyendo nexos sociales que rebasan las fronteras nacionales. Es también una modificación de las estructuras de dichas relaciones". ${ }^{13}$

Caglar, ofrece una nueva óptica que permite hacer visible la creciente intensidad y extensión de los flujos circulares de personas, bienes, información y símbolos alcanzados por las migraciones internacionales. Permite analizar cómo los migrantes construyen y reconstruyen sus vidas de forma simultánea en más de una sociedad [Caglar, 2001: 607].

Guarnizo, lo define como "el proceso de la migración internacional desde las implicaciones de las teorías asimilacionistas y la perspectiva transnacional.Las teorías asimilacionistas se enfocan en la sociedad receptora y en los procesos que afectan a la población y a la cultura autóctona, la perspectiva transnacional

12 Portes, A. Convergencias teóricas y evidencias empíricas en el estudio del Transnacionalismo de los inmigrantes. Migración y Desarrollo,2005, primer semestre, p. 2-19.

13 Memorias de seminario sobre migración internacional colombiana y la conformación de comunidades transnacionales, Colombia Nos Une, Ministerio de relaciones exteriores, 2003. involucra la interacción entre destino y origen, poniendo énfasis en este último". ${ }^{14}$

Held concluye que la situación migratoria debe entenderse como "un conjunto de procesos, no como una condición única, reflejando la emergencia de redes interregionales y sistemas de interacción e intercambio. Así, se logra una interconectividad global y transnacional que construye complejas redes de relaciones entre comunidades, estados, instituciones internacionales, organizaciones no gubernamentales y corporaciones multinacionales que constituyen el orden mundial". ${ }^{15}$

Ahora bien, las comunidades que se forman a partir de los movimientos transnacionales se caracterizan por una situación particular: los migrantes, aquellos que se quedan en el país de origen y los nacionales de los países anfitriones están conectados por lazos sociales y simbólicos densos y fuertes. Esos lazos, teniendo como base la solidaridad, configuran a través del tiempo y el espacio redes y circuitos en los países de origen y de destino [Faist, 1999: 10].

Con lo anterior, se comienza a formar un nuevo concepto, el de transmigrante. En este caso la migración entendida como "el movimiento del hombre de un lugar de residencia a otro" ya no es la situación excepcional en la vida; se convierte en una forma de viviry de sobrevivir en sí misma.

De esta manera, al vivir a través de las fronteras, los transmigrantes forman parte de los procesos de construcción social de dos o más naciones. Se da así una

14 Tomado de www.ub.es/geocrit/b3w-667.htm 15 Held et. al. (1999: 27). Held, D., A. McGrew, D. Goldblatt, and J.Perraton.(1999).Global Transformations: Politics, Economics and Culture. Polity and Stanford University Press. 
confrontación entre los migrantes y las características hegemónicas - como raza y etnicidad- de la sociedad de acogida, las cuales son intrínsecas a ésta y forman parte de los mencionados procesos de construcción social [Gledhill,1998].

Estas relaciones se mantienen a través del traslado físico continuo de los individuos entre sus áreas de salida y de destino. Sin embargo, no todos los inmigrantes son transmigrantes; sólo lo son aquellos inmigrantes que comparten dos culturas, tienen hogares en dos países y construyen su vida por medio de intercambios continuos a través de fronteras nacionales.

Los cambios tecnológicos y socioculturales inducidos por los procesos de globalización influyen sin duda en la naturaleza de las migraciones al dotarlas de un marcado carácter transnacional.De ahí que, como señala Bauböck [1998,26], resulte ineludible "considerar la migración como un fenómeno genuinamente transnacional, no sólo en el momento de cruzar las fronteras, sino también con respecto a las filiaciones sociales resultantes."

Es en esta teoría donde encontramos tres tipos de espacios sociales transnacionales que surgen de la migración internacional [Faist, 2000: 203]. (1) Grupos transnacionales de parentesco: cuyo recurso primario de las uniones es la reciprocidad, que está caracterizada principalmente por la premisa "lo que una parte recibe de otra requiere algún tipo de retorno",y tiene como objetivo el mantenimiento de las normas sociales. En otras palabras, se busca el control sobre los miembros de los grupos pequeños. Un ejemplo para esta situación serían las remesas desde el hogar o de los miembros de la familia desde el país de inmigración al país de emigración (trabajadores migrantes).(2) Circuitos transnacionales: cuyo recurso primario de las uniones es el intercambio, cuya premisa "obligaciones mutuas y expectativas de los actores; resultados en forma de actividad instrumental", se caracteriza principalmente por la utilización de las ventajas del que está adentro como el idioma, redes sociales fuertes y débiles entre cercanos, etc. Las redes de comercio en el Líbano, China e India son un buen ejemplo. (3) Comunidades transnacionales: cuyo recurso primario de las uniones es la solidaridad, caracterizada por ideas compartidas, creencias, evaluaciones y símbolos, expresadas como forma de una identidad compartida que moviliza representaciones colectivas en forma de uniones simbólicas. Ejemplo: religión, nacionalidad, etnicidad, diásporas (Judíos,Armenios, Palestinos, Kurdos).

\section{ii. Descripción histórica del Valle del Cauca}

El territorio vallecaucano, ubicado entre la cordillera occidental y la cordillera central,cuenta con una de las conexiones más estratégicas hacia el puerto de Buenaventura, principal punto de conexión al Océano Pacifico con Colombia. El Valle del Cauca se caracterizó por ser uno de los primeros departamentos de Colombia que presentó un jalonado desarrollo agroindustrial en sus cultivos desde inicios del siglo,lo que le permitió abastecerse de una rica historia social, política y económica.

El Departamento del Valle del Cauca, fue creado por medio del Decreto 340 del 16 de abril de 1910 [Londoño, 2008]. Fue un departamento consolidado por diferentes actores inmersos en su historia: desde los migrantes de tierras aledañas, 
como pobladores extranjeros, quienes, al observar las virtudes de las tierras del Valle, decidieron establecer sus inversiones en él.Los fértiles y extensos terrenos del Departamento del Valle del Cauca le permitieron caracterizarse como una tierra apta para el crecimiento agroindustrial. La caña de azúcar, junto a otros cultivos como la quina, el tabaco, el café y el arroz, junto con el aprovechamiento de los pastizales para la ganadería, fueron consolidando la economía del Departamento como una de las más destacadas por su rápido y avanzado crecimiento en el país. Estos hechos se vieron reflejados en las políticas del Gobierno Nacional cuando este tomó la decisión para mediados del siglo XIX de consolidar el cultivo de la caña como fuente principal de riqueza y empleo para los diversos pobladores de la región vallecaucana [Motta,2007].

No obstante, atado al fortalecimiento de la agroindustria, ${ }^{16}$ fue muy notorio, tanto en el Departamento como en todo el país, el surgimiento del fenómeno de la violencia. En términos generales, pueden enumerarse como causas de ese surgimiento a la expansión industrial, la desigual distribución de tierras, el fortalecimiento de las empresas multinacionales en el Departamento, entre otras. Estos fueron factores que incidieron en una crisis de credibilidad en el país, y para los pobladores del mismo, cuyo principal efecto fueron fuertes olas de desplazamientos internos, en su mayoría hacia el Departamento del Valle del Cauca. Todo este desequilibrio permitió el afianzamiento del narcotráfico y las

16 Kruijt Dirk (1982) - Industrialización y desarrollo regional en Colombia. fuerzas revolucionarias a partir de 1970 en Colombia.

Es así como, continuando con la idea anterior, el narcotráfico y el conflicto collevaron a que se precipitara la búsqueda de mejores índices de seguridad y sostenibilidad por parte de los colombianos. Esa búsqueda se reveló en el hecho de que cada vez más compatriotas, especialmente los vallecaucanos, fueran en pro de una alternativa que les garantizará mejores condiciones de vida.

La migración fue un derivado de la creciente desigualdad, inequidad y falta de autoridad por parte del Estado,y fueron el desarraigo y la falta de expectativas de superación de la violencia los que impulsaron a la población a salir en búsqueda de nuevas opciones.Así,la migración hacia el exterior, cuyos casos aumentaron dramáticamente, se consolidó como una de las alternativas más inmediatas y posibles para salir de esa situación tan precaria.

La migración internacional se presentó para el territorio colombiano en tres oleadas. La primera, que tuvo lugar entre 1965 y 1975, fue hacia Venezuela, Estados Unidos, Ecuador y Panamá, donde la mayoría de los colombianos provenían de los grandes centros urbanos como Bogotá,Medellín,Cali,Eje Cafetero,entre otros. Luego, para los años 80, encontramos la migración hacia Venezuela, por el gran auge petrolero del momento. Por último, se tiene una oleada mucho más diversificada desde los años 90 hacia Europa, Canadá y Australia, entre otros, la cual, hasta el día de hoy, se presenta hacia nuevos destinos, antes no tenidos en cuenta. El flujo migratorio se ha ido diversificando a tal grado que ha sido ne- 
cesaria una mayor atención por parte del Estado colombiano [CONPES 3603,2008:31]. La violencia y sus derivados, se convirtieron en las causas más determinantes para que se presentara el fenómeno de la migración interna e internacional en Colombia, y, en este caso, con mayor presencia en el Valle del Cauca. En la actualidad, sin embargo, las personas que emigran desde el Valle del Cauca lo hacen, a su vez, impulsados por la integración de matrimonios y reunificación familiar. Las motivaciones han ido cambiando a través del tiempo basadas en la historia económica,social y política del momento.

"Para los años 70 vivían fuera del país 216.900 colombianos, para los 80 había más de medio millón.Pero se calcula que entre 1991 y 2001, una cifra parecida sale del país y no regresa [Beltrand,2003]; y que en la actualidad,según datos del censo del DANE 2005 entre tres y cuatro millones de colombianos residen en el exterior". ${ }^{17}$

En los datos encontrados en la encuesta realizada por el Departamento Administrativo Nacional de Estadística (DANE) para 2005, se evidencia que el Valle del Cauca, es el Departamento con el mayor nivel de pobladores en el exterior, con un $24.1 \%$, seguido de Bogotá con un $18.7 \%$,Antioquia con $11.9 \%$, Risaralda con $7.8 \%$ y Atlántico con 6.6\%.

Según la Encuesta Nacional sobre Migraciones Internacionales y Remesas (ENMIR), sobre la encuestas del DANE en el 2005 y la Encuesta a Beneficiarios de Remesas en agencias de Instituciones Cambiarias EBRIC 2004, se estima que existe una población aproximada de 22.000 receptores de remesas. Precisamente, esta es otra de las fuertes circunstancias por las que deben plantearse estrategias

17 Tomado de www.migracionescolombianas.edu.co de sostenibilidad para las familias que dependen de los dineros enviados por sus familiares ubicados en el exterior.

América España Solidaridad y Cooperación (AESCO), en su última encuesta realizada en Julio del 2009, arrojó que el municipio con mayor nivel de migración, del Valle del Cauca, fue Cali (Ver tabla No.1), donde las comunas 19, 02, 17 y 10 (Ver tabla No.2) fueron las que presentaron mayores experiencias de migración en sus hogares. ${ }^{18}$

Por otro lado, se observa que un gran porcentaje de familias que viven de las remesas que les envían personas domiciliadas en el exterior.También se puede observar a través de las causas por las que se han ido tornando la decisión de migrar en el Departamento, donde ya no solo la necesidad económica es la determinante.

La caracterización de la cultura vallecaucana y,en general,la colombiana,deja en evidencia el apego por la cultura y los estrechos vínculos con la que los connacionales conviven en sus países de destino, todo esto a causa de las situaciones que los llevaron a tomar la decisión de migrar. La teoría trasnacional dentro de sus múltiples conceptualizaciones es la que mejor interpreta la irrompible conexión que los migrantes mantienen con sus familiaresy allegados (Memorias del Seminario sobre migración internacional colombiana y la conformación de comunidades transnacionales, Colombia Nos Une, Ministerio de relaciones exteriores, 2003).

\section{iii. CONPES y política departamental}

El Consejo Nacional de Política Económica y Social CONPEs fue creado por

18 Asociación América España solidaridad y cooperación AESCO COLOMBIA, 
Tabla No. 1. Relación de los municipios del departamento del Valle del Cauca, con hogares con emigrantes en el exterior.

\begin{tabular}{|c|c|}
\hline Municipios Del Valle & $\begin{array}{c}\text { Hogares Con Emigrantes } \\
\text { En El Exterior }\end{array}$ \\
\hline Cali & 36.984 \\
\hline Buenaventura & 3.373 \\
\hline Guadalajara De Buga & 2.529 \\
\hline Cartago & 3.468 \\
\hline Jamundi & 1.039 \\
\hline Palmira & 7.924 \\
\hline Tulua & 3.655 \\
\hline Yumbo & 1.598 \\
\hline
\end{tabular}

Fuente: Datos DANE 2005 hogares con experiencia migratoria por departamentos y municipios. Elaborada por el Comité de Investigaciones AESCO- COLOMBIA Julio de 2009

Tabla No. 2. Relación de las comunas del municipio de Santiago de Cali, con número de hogares con experiencia migratoria.

\begin{tabular}{|c|c|}
\hline Comuna & Barrios \\
\hline 6 & $\begin{array}{c}\text { Petecuy I, II, y III etapa, Floralia I, II, y } \\
\text { III etapa, Quintas de Salomia, Solares de } \\
\text { Comfandi. }\end{array}$ \\
\hline 7 & $\begin{array}{c}\text { Alfonso López I, II, y III etapa, Andrés } \\
\text { Sanín, Los Pinos, La Ceiba, Cali Bella. }\end{array}$ \\
\hline 8 & $\begin{array}{c}\text { Chapinero, La Floresta; Las Américas, } \\
\text { Primitivo Crespo, Santa Fe, Uribe Uribe, } \\
\text { Villa Colombia. }\end{array}$ \\
\hline 9 & Barrio Obrero, Bretaña, Guayaquil, Junín. \\
\hline 10 & $\begin{array}{c}\text { Cristóbal Colon, El Guayabal, La Selva, Las } \\
\text { Acacias, Olímpico, Pasoancho. }\end{array}$ \\
\hline 11 & $\begin{array}{c}\text { La Gran Colombia, José Holguín Garcés, } \\
\text { Los Conquistadores. }\end{array}$ \\
\hline 13 & Calipso, El Diamante, El Vergel, Lleras \\
Restrepo, Marroquín.
\end{tabular}

Fuente: Datos DANE 2005 hogares con experiencia migratoria por departamentos y municipios. Elaborada por el Comité de Investigaciones AESCO- COLOMBIA Julio de 2009 
la Ley 19 de 1958. Este organismo es la máxima autoridad en materia de planeación y se desempeña como cuerpo asesor del Gobierno en todos los aspectos relacionados con el desarrollo económico y social del país. Para lograrlo, coordina y orienta a los organismos encargados de la dirección económica y social en el Gobierno, a través del estudio y aprobación de documentos sobre el desarrollo de políticas generales, que son presentados en sesión [Departamento nacional de planeación. República de Colombia.www. dnp.gov.co/conpes.aspx].

Específicamente, el documento coNPES 3603 publicado en agosto 2009 está dedicado a un estudio detallado acerca de la creación de la Política Integral Migratoria (PIM) del Gobierno Nacional, donde se presentan los lineamientos, estrategias y programas que el gobierno aborda bajo las diferentes situaciones, ya sean económicas, sociales, políticas, educativas y culturales, de la población migrante internacional. Por otro lado, analiza los diferentes cambios institucionales que se han dado para poder llevar a cabo correctamente la PIM. Por último, describe las actividades, el presupuesto y las recomendaciones para cumplir los objetivos propuestos bajo esta política. Dentro de sus principales objetivos se encuentran: 1) dar un tratamiento integral a cada una de las dimensiones de desarrollo ${ }^{19}$ de esta población, y 2) mejorar la efectividad de los instrumentos utilizados para la implementación de estrategias y programas referentes a la población migrante, así como los objetivos de política formulados en el Plan Nacional de Desarrollo

19 Dimensiones económicas, políticas, sociales, culturales y educativas.
2006-2010 “Estado Comunitario: Desarrollo para Todos" [Documento conPEs 3603, Política Integral Migratoria, República de Colombia, http://colombiaemb.org/docs/ CONPES\%203603\%20-\%20Poltica\%20Integral\%20Migratoria.pdf, agosto de 2009].

El documento comienza con un marco jurídico desarrollado por anteriores gobiernos frente al fenómeno migratorio internacional, donde se estudiaron tratados internacionales ratificados por Colombia, ${ }^{20}$ instrumentos jurídicos de orden nacional ${ }^{21}$ y diversas organizaciones institucionales, ${ }^{22}$ así como antecedentes en materia de política pública frente al tema migratorio, para el período comprendido entre 2002-2008. Dentro de los programas estudiados se encontraban:

- El programa Colombiano Seguro en el Exterior,desarrollado por el Ministerio de Relaciones Exteriores, el Instituto de Seguros Sociales iss y la empresa Giros y Finanzas S.A., cuyo representante legal en Colombia es Western Union.

- El portal Colombia Aprende, organizado por El Ministerio de Educación Nacional, en conjunto con la Red Latinoamericana de Portales Educativos (RELPE) y la Fundación Telefónica.

- Becas y créditos educativos para estudios en el exterior, programa educación

20 Convención internacional sobre la protección de los derechos de todos los trabajadores migratorios y de sus familiares. [Res.ONU 45/158 de 1990] / Decisión 545 de la CAN / Decisión 583 de la CAN Sustitución de la Decisión 546 / Ley 450 de 1998 / Ley 479 de 1998 / Ley 968 de 2005

21 Constitución Política [Art.9,24,96,97,100,189,227,289, 337 y el Preámbulo] / Ley 76 de 1993 y Ley 991 de 2005 22 Decreto 1974 de 1995 / Decreto 2482 de 1994 / Resolución 0326 de 2001 del Ministerio de Relaciones Exteriores / Decreto 1239 de 2003 / Resolución 3131 de 2004 del Ministerio de Relaciones Exteriores / Decreto 110 de 2004 / Decreto 2622 de 2009 / Decreto 2884 de 2008 
técnica, con apoyo del Instituto Colombiano de Crédito y Estudios Técnicos en el Exterior (ICETEX).

- Proceso de convalidación de títulos, controlado por el Ministerio de Educación y la Comisión Nacional Intersectorial de Aseguramiento de la Calidad (CONACES)

- Plan comunidad en el exterior y el portal Redes Colombia, creados para promover las asociaciones de las comunidades colombianas en el exterior en apoyo en coordinación con los consulados de Colombia.

- Servicio consular,servicios consulares virtuales y consulados móviles, creados con el fin de agilizar los diferentes procesos consulares.

- Creación de diversos programas en contra de la trata de personas, como el Comité Interinstitucional para la Lucha contra la Trata de Personas.

- Programa Colombia Nos Une, con el cual se pretende promover los vínculos entre colombianos que están fuera y sus familias que quedaron en el país, sus regiones de origen y de manera general con Colombia

Siguiendo con el análisis, el documento explica cinco principios que reconoce la PIM, los cuales corresponden a:

1. Principio de Coherencia o Reciprocidad, a través del cual el Estado colombiano se compromete a actuar de acuerdo con los tratados internacionales frente al tema de la migración respecto a las dinámicas internacionales.

2. Principio de Integridad y Largo Plazo, donde la PIM deberá ajustarse a los diferentes cambios coyunturales políticos, económicos y sociales.
3. Principio de Concordancia, donde la PIM debe estar desarrollada de acuerdo a la política exterior,de esta manera servirá de apoyo llegado el momento de participar en tratados y acuerdos internacionales.

4. Principio de Plena observancia de garantías individuales, para lo cual, a todo colombiano, se le reconocen sus derechos fundamentales tanto fuera como dentro del país.

5. Principio de Focalización, con el cual se procura atender con prioridad a aquellos migrantes más vulnerables por su condición dentro del país donde se encuentra.

El CONPES 3603, desarrolla tres tipos diferentes de Migración internacional,la circular, la temporal y la internacional. ${ }^{23}$ El documento gubernamental,manifiesta que el Migrante Internacional corresponde a toda persona que cambia de país en el que tiene su residencia habitual,y que esto puede darse durante un período largo o corto de tiempo-inferior a doce meses.

Por otro lado, es menester afirmar que existe una limitada atención a los fenómenos migratorios por parte del Estado colombiano, este es el mayor problema encontrado dentro del estudio realizado. La desatención a estos temas se evidencia, en primera instancia, por los instrumentos utilizados para la implementación de las acciones y estrategias a favor de la población migrante ya que no han sido efectivos y, en segundo término, en que

23 La Migración Circular, es decir,cuando el migrante va y vuelve al país de acogida por ciertos períodos. ConPES 3603 pg26; la Migración Temporal, situación en la que el migrante va por un período a un país extranjero. CONPES 3603 pag26; la Migración Internacional, o el movimiento de nacionales hacia un país diferente al de nacimiento para establecer allí su residencia habitual. CONPES 3603 pag26. 
las dimensiones de desarrollo de la población migrante no han sido atendidas de manera oportuna y completa.

La primera evidencia viene dada por la estructura institucional limitada del Estado colombiano, la deficiente calidad de la información sobre la población migrante que obtiene el mismo, y a que la información no se encuentra actualizada ni centralizada. Un caso que ejemplifica la falta de atención al respecto es el del servicio consular de carrera, que apenas está en proceso de consolidación en Colombia y que cuenta con escasos recursos en información y personal capacitado para acudir a los migrantes.

La segunda evidencia se origina en el problema de la trata y tráfico de personas, esta situación es una forma de esclavitud en la que se degrada al ser humano a la condición de objeto, que se negocia en cadenas mercantiles, se traslada dentro o fuera del país, para luego, en el destino final, ser sometido a condiciones de explotación u otros fines ilícitos. Es una violación a los derechos humanos que atenta contra la esencia misma del hombre al reducirlo a forma de objeto. El Estado ha limitado su intervención al respecto.

El negocio de la trata de personas es operado por redes de traficantes nacionales y transnacionales que actúan ilegalmente para obtener beneficios económicos, reclutan víctimas por cualquier método, entre los que figuran los ofrecimientos de mejorar su calidad de vida [Fundación Esperanza,el tráfico de personas, Naufragio de Sueños, Ramos López Ltda., Colombia, 2003,pg.15].

Lo anterior ocurre como consecuencia de la baja participación cívica y comunitaria de las asociaciones de colombia- nos en el exterior, identificadas por su registro en los consulados más cercanos a su lugar de residencia y en las redes creadas en Redes Colombia del Ministerio de Relaciones Exteriores, la ausencia de mecanismos para la generación de vínculos entre los alumnos colombianos de educación superior migrantes y el sistema educativo colombiano, al igual que mecanismos para la generación de vínculos entre los estudiantes, docentes y/o investigadores colombianos en el exterior y la comunidad educativa en Colombia, la falta de información para la convalidación de títulos de educación superior en el exterior, así como de títulos extranjeros en Colombia, la inserción laboral precaria de los migrantes, el bajo acceso a sistemas de protección social,y diagnósticos insuficientes para la definición de acciones en materia de género y familia [Fundación Esperanza, el tráfico de personas, Naufragio de Sueños, Ramos López Ltda., Colombia, 2003, pg.15].

Otro tema importante que se trata dentro del documento conPEs es el aporte que hacen las remesas ${ }^{24}$ a la economía nacional, ingresadas bajo un registro y otras sin identificar. Sin embargo, desde los últimos tres años el comportamiento de las remesas ha sido decreciente, pero no deja de aportar al Producto Interno Bruto (PIB) a través de la balanza de pagos. Por lo que Colombia es el tercer país que más remesas recibe en América Latina,

24 La remesa es una operación por la cual se manipulan por parte de los bancos, según las instrucciones recibidas en cuanto a importe, plazo y divisa, los documentos financieros (letras de cambio,pagarés, cheques, recibos de pago)y no financieros (facturas, documentos), con el fin de entregarlos contra la aceptación y/o pago, o según otros términos y condiciones. Banco de la república www.banrep.com.co 
después de México, Brasil y El Salvador. Ahora bien, si reconocemos el tamaño de estos dos países y sus economías, la diferencia con nuestro país salta a la vista.

El objetivo central del proyecto de ley de la Política Integral Migratoria (PIM) busca garantizar una atención suficiente, efectiva y coordinada sobre todas las dimensiones de desarrollo de la población colombiana en el exterior y los extranjeros residentes en el país, con lo cual se busca dar respuesta a la limitada atención prestada por parte del gobierno al fenómeno migratorio, para la implementación de estrategias y metas concretas ya planteadas. Se tiene por objetivos específicos, precisamente, aquellos que ayuden a solucionar los ejes problemáticos planteados anteriormente, para lo cual se generaran lineamientos institucionales y organizacionales para el trabajo en conjunto que también ayudará en la actualización de los sistemas de información. También se busca alcanzar la plena implementación de las acciones propuestas sobre cada una de las dimensiones de desarrollo de la población migrante (seguridad, participación cívica y comunitaria, educativa, cultural, social y económica).

El plan de acción a seguir, busca fortalecer la Comisión Nacional Intersectorial de Migración ${ }^{25}$ (CNIM), a través de la recomendación de sus entidades miembros para el desarrollo de la PIM. El mejoramiento de la información sobre la migración internacional, se logrará a través de asistencia técnica que brinda el DANE con la Encuesta de Colombianos Residentes

25 Órgano para la coordinación y orientación en la ejecución de la política migratoria del país, creada bajo el decreto 1239 de 2003. Ministerio de Relaciones Exteriores en el Exterior (ECRE), el fortalecimiento del servicio jurídico con los consulados móviles, y para las dimensiones antes mencionadas, en cuanto a la seguridad humana se trabajará con el Comité Interinstitucional de Lucha contra la Trata de Personas y la línea gratuita contra la trata de personas, en este sentido se apoyarán las investigaciones académicas que hagan referencia a este tema; desde la dimensión educativa, se trabajará de la mano con el portal Colombia Aprende, ${ }^{26}$ se establecerán mecanismos de coordinación a través de la utilización de redes interinstitucionales como la Red Nacional Académica de Tecnología Avanzada ${ }^{27}$ (RENATA), Red Clara, ${ }^{28}$ internet $2^{29}$ entre otros, además de divulgar ampliamente el portafolio de servicios prestados por el ICETEX.

Desde la dimensión cultural,el programa Colombia Nos Une ${ }^{30}$ y el Ministerio de Cultura, producirán materiales de apoyo

26 Colombia Aprende es el portal educativo del Ministerio de Educación Nacional, creado el 24 de mayo de 2004, como un proyecto estratégico dentro del Programa Nacional de Uso de Medios y Nuevas Tecnologías de la Información y la Comunicación del Plan Sectorial “La Revolución Educativa 2002-2006”. 27 RENATA es la red de tecnología avanzada que conecta, comunica y propicia la colaboración entre la comunidad académica y científica de Colombia con la comunidad académica internacional y los centros de investigación más desarrollados del mundo.

28 La visión de CLARA es ser un sistema latinoamericano de colaboración mediante redes avanzadas de telecomunicaciones para la investigación,la innovación y la educación. CLARA desarrolla y opera REDCLARA, red de Internet avanzada que se estableció en América Latina -para la interconexión regional- en el año 2004 29 Es una red de cómputo sustentada en tecnologías de vanguardia que permiten una alta velocidad en la transmisión de contenidos y que funciona independientemente de la Internet comercial actual 30 El Gobierno Nacional dispuso en el Plan Nacional de Desarrollo 2003 - 2006, adelantar una política integral relacionada con los diversos grupos que conforman la comunidad colombiana en el exterior,con el propósito 
"Si los gobiernos defienden la libertad de circulación del capital, de bienes y de ideas, es poco probable que sean capaces de frenar la circulación de personas"

a las redes sociales de colombianos que ayuden a mantener sus vínculos con tradiciones del país. Respecto a la seguridad social, a través del portal Redes Colombia se difundirá información relacionada con el acceso y la permanencia al sistema de Seguridad Social en Colombia de aquellas personas que están fuera de la nación. Para la dimensión económica,se desarrollarán estrategias para el aprovechamiento de las remesas a través del Departamento Nacional de Planeación, para así también evitar el problema de lavado de activos.

El CONPES 3603, finalmente, corresponde a un documento que encierra todo un trabajo realizado en conjunto por instituciones gubernamentales y no gubernamentales - nacionales e internacionales- que busca unir esfuerzos para mostrar, más que cifras, los factores causales del fenómeno migratorio en Colombia. Dentro del análisis que presenta el documento, lleva implícito el hecho de que los movimientos de masas se dan por diversos factores que, como menciona Carassou, pueden ser voluntarios o no. Es así como las motivaciones pueden ser políticas, económicas, sociales o pueden ser incitadas por la búsqueda de mejores oportunidades que eleven el nivel de vida

de estimular sus vínculos con el país y hacerlos objeto de políticas públicas. del posible migrante. Es por ello que este documento, desde su estructura, se ha preocupado por analizar el fenómeno migratorio internacional desde diferentes dimensiones, cuenta con una visión holística, de esta manera el Gobierno Nacional pretende comprender cada faceta pero encuentra precisamente que siguen presentándose inconvenientes que no permiten el desarrollo eficiente de los diversos programas propuestos.

Por otro lado, el proceso de modernización y los nuevos aportes tecnológicos, [Meyer, 2006:90-125pg], han facilitado la comunicación entre la población migrante y sus familias, vínculos propios de los cuales no pueden desligarse, de los cuales nacen conceptos como el transnacionalismo, que dan nombre propio a las relaciones que se mantienen entre el migrante, su cultura, sus características y tradiciones. Los portales de entidades como Colombia Nos Une, los programas que realizan las diferentes embajadas con el fin de conmemorar fechas especiales de Colombia, para mantener vivas muchas de nuestras costumbres, hacen parte de algunas de las opciones por las cuales los colombianos en el exterior no pierden contacto con su país.Sin embargo, lo anterior es posible gracias a entidades, como el DANE, que se preocupan por desarrollar encuestas, como la ECRE (Encuesta de Colombianos Residentes en el Exterior), que permiten conocer más de cerca las necesidades de los colombianos residentes fuera de su nación, y de esta manera, el Gobierno Nacional, o entidades preocupadas por el tema migratorio, pueden aplicar proyectos con mayor implicación sobre aquellas variables de mayor importancia para el migrante. 
La PIM muestra, dentro de sus principios, una tendencia a analizar la situación migratoria como todo un conjunto de procesos, donde hacen parte tanto las redes interregionales como diversos sistemas de interacción, por lo que se está llevando a cabo como argumenta [Held et. al. 1999:27] "una ínterconectividad global y transnacional que construye complejas redes de relaciones entre comunidades, estados, instituciones internacionales, organizaciones no gubernamentales y corporaciones multinacionales que constituyen el orden mundial", dado que tanto el principio de coherencia, de integridad y largo plazo, de concordancia, de plena observancia de garantías individuales y de focalización, muestran una relación directa con el trabajo que debe realizarse en conjunto con las instituciones internacionales, y no gubernamentales para permitir el acoplamiento al fenómeno internacional migratorio que se está presentado en el mundo; además, en este proceso de globalización y generación de comunidades transnacionales, como explica Sassen, “el Estado se globaliza, especialmente el Poder Ejecutivo, pero la Legislatura se domestica”, por lo que el planteamiento de la PIM es, básicamente, el resultado de una legislación nacional que busca el apoyo y la cooperación internacional para el tema global de la migración.

iv. Relación de las instituciones con la política pública de la migración del Valle del Cauca.¿Cómo influyen?

Dentro del proceso investigativo y trabajo de campo, se realizó un contacto directo con representantes de diferentes instituciones que trabajan el tema de la migración internacionaly de las cuales se provee la información para el desarrollo de documentos de carácter político,social y cultural,por parte de la Gobernación del Valle del Cauca.

Conociendo la situación de la migración internacional en el Valle del Cauca, es claro dar razón de que el tema de los connacionales en el exterior fue de gran relevancia desde inicios del siglo XXI, según el Censo del año 2005 realizado por el DANE, donde el Departamento del Valle del Cauca mostró el mayor porcentaje de migrantes colombianos.

Fue bajo la dirección del gobernador Juan Carlos Abadía Campo,quien junto al Secretario de Gobierno, un grupo de apoyo y el respaldo y la experiencia de más de 14 años de la Fundación Esperanza, permitieron dar creación al documento legal de la ordenanza No.270 del 9 de diciembre del 2009. En él quedó establecido la consolidación de un equipo de trabajo especializado en la búsqueda de soluciones en torno al tema de la migración. El Consejo Departamental de Atención Integral para la Migración, en proceso de formación hasta la fecha (2009), del Departamento del Valle del Cauca a cuyo cuidado estaría la formulación, seguimiento, desarrolloy coordinación de programas y proyectos enmarcados en el tema migratorio. ${ }^{31}$

Este consejo está conformado por 21 miembros, ${ }^{32}$ entre ellos entidades gu-

31Gobernación del Valle del Cauca, 2008, Ordenanza no. 270 del 17 de diciembre de 2008, http://www.valledelcauca.gov.co/asamblea/publicaciones.php?id=4806. 32 Miembros del Consejo departamental de atención integral para la migración: El Gobernador(a) o su delegado. La Secretaría de Gobierno, quien ejercerá la coordinación técnica. La Secretaría de Desarrollo Social. La Secretaría de Educación. La Secretaría de Equidad de Género para las Mujeres Vallecaucanas. La Secretaría de Planeación. La Secretaría de Salud 
bernamentales y no gubernamentales, quienes tienen a su cargo la creación de espacios que garanticen el pleno goce de los derechos humanos de los migrantes. Es decir, que son funciones o actividades que ya se vienen trabajando desde diferentes entidades con mucha anterioridad.El fenómeno de la migración internacional es antiguo, pero es sólo a partir del 2008 cuando los entes territoriales toman una posición dentro de la situación por la que viven los colombianos en el exterior. Es por ello que se evidencia la necesidad de resaltar el papel que han desempeñado cada una de las entidades, nacionales e internacionales, dentro del marco de los Derechos Humanos y Derechos Internacionales de los colombianos en el exterior $\mathrm{y}$ de las migraciones en general.

La Fundación Esperanza en su trabajo a través de los años, ha canalizado su búsqueda comprometida con los derechos humanos de la población en contextos de migración, trabajando en torno al tema de la migración internacional y los efectos que ella trae a las familias que tienen alguna relación con este tema. Su prioridad es aquellos temas en los que es vital la asistencia por parte de las entidades gubernamentales, realizando Municipal y Departamental o su Delegado. El (la) Director (a) de Bienestar Familiar.El Director del DAS o su Delegado. El Director del Sena o su Delegado. Un Delegado de la Defensoría del Pueblo. El Personero Municipal de Cali o su Delegado. Un Miembro de la Asociación Departamental de Personeros.Un Delegado de la Procuraduría. Un Representante de la Cámara de Comercio de la ciudad capital. Un Representante de la Universidad Pública y un Representante de las Privadas. Tres Representantes de las ONGs que trabajen en el tema.Un Representante de las filiales de las centrales de trabajadores del Valle del Cauca. Representantes de la Pastoral de la movilidad humana.Un Representante de la Red de Vallecaucanos en el Exterior. Un Delegado de la Pastoral Cristiana. diversas apariciones que dejan claro su interés por defender y exaltar el cuidado que necesitan los migrantes colombianos en el exterior.

La Fundación Esperanza trabaja en seis líneas de intervención: (1) las líneas de estudios e investigaciones (2) Campañas de sensibilización,(3) Un Sistema de Atención al Migrante SAM, y su consultorio jurídico y social, (4) Iniciativas de políticas públicas, locales, regionales y nacionales (5) Iniciativas de migración y desarrollo local (6) Migración, infancia y familia,y Explotación sexual y comercial infantil.

Todo su trabajo ha ido en búsqueda de canales que permitan la consolidación de estrategias de conexión que los connacionales en el exterior puedan tener para con su país de origen. Su búsqueda radica en los lazos de protección que puedan tener ellos, desde su lugar de origen,y en cómo el Estado puede proveerles mejores herramientas de sobrevivencia y desarrollo.

De igual manera, la Fundación Esperanza, ha realizado un trabajo de investigación muy intensivo, en conjunto con el Observatorio Colombiano de Migraciones, (ocM), y la Red Alma Mater. El estudio se materializa con la Encuesta Nacional de Migración Internacional y Remesas, (ENMIR) realizada en Norte, Centro Occidente -Antioquia y Eje cafetero-Valle del Cauca y Bogotá,y que es un trabajo que permite avanzar en el análisis que sobre los colombianos en el exterior.La encuesta ha sido apelada en consideración a partir del censo realizado en el 2005 por el DANE, ya que no solo evidenció nuevos aspectos que hasta el momento no se tenían en cuenta sino que contradijo cierta infor- 
mación de la que se tenía como base en el tema de la migración para Colombia.

Los aportes de esta investigación de campo y el gran volumen de investigaciones y análisis realizados por la fundación, ha permitido expandir y crear una mayor conciencia sobre las situaciones y consecuencias que se derivan del tema de la migración internacional.

Por otro lado, se encontró que la Comisión Europea de Ayuda al Refugiado (CEAR), una organización no gubernamental que trabaja hace 30 años en España, realiza una coordinación dirigida por el gobierno de ayuda al migrante.Ésta inició sus servicios con la prestación jurídica a migrantes en España de gran utilidad para los vallecaucanos. ${ }^{33}$ CEAR viene trabajando en Colombia hace aproximadamente un año. Su actuación dentro del país, se vio impulsada luego de un estudio realizado en España sobre los connacionales que llegaban como refugiados a ese país. Con respecto a los colombianos se distinguieron dos situaciones, una de ellas es la falta de información, o información errada, con la que el colombiano sale del país. Lo que provoca la existencia de una gran red de tráfico de personas. Por, otro lado, se detectó una gran red de tráfico, de documentación falsa, a través de la cual les vendían paquetes de denuncias y documentación a las personas a cambio de asilo político en España por una suma de varios millones, lo que provocaba una disminución en la credibilidad que las organizaciones que trabajan en búsqueda de la protección de las personas que vienen bajo esta figura, como la CEAR.

33 La información recibida por parte de la organización fue bajo el testimonio de la trabajadora social,de la oficina de CEAR en la ciudad de Cali,7 de octubre del 2009.
Específicamente, el tema del tráfico de personas por desinformación y por documentación falsa, presentó una fuerte presencia en el Valle del Cauca y, a raíz de que el tema del asilo es tratado con gran cuidado en España especialmente en el aeropuerto de Barajas donde se les recibe y se les hace la revisión de todos los documentos que llevan soportando la realidad del suceso, el Estado español decidió realizar un proyecto liderado por la CEAR, donde proveen de información gratuita a todas las personas que tengan intención de migrar a todos los países y en especial a España. Buscando con ello crear una mayor conciencia del riesgo que tiene la falsificación de documentos ante una futura protección por parte de la Unión Europea.

El tema de la migración y en especial el asilo, es uno de los de mayor relevancia para la Comisión Europea, y es por esto que cuenta con muchas entidades no gubernamentales encargadas de tratar y apoyar a los migrantes que están bajo esta figura por medio de varias líneas de soporte y apoyo en el tema de la migración. Es así como la CEAR buscó la forma de tener un contacto con las entidades encargadas del tema de asilo desde Colombia,y en especial en el Valle del Cauca. Realizó un estudio de estas entidades y no se encontró con mayores resultados pues el tema de la migración internacional desde el Valle del Cauca aún no estaba dentro de la agenda del Departamento sino hasta apenas el 2008.

Bajo la Gobernación de Angelino Garzón, se puso el tema de las migraciones internacionales sobre el tapete y se comenzaron a crear conexiones de apoyo con la Universidad Santiago 
de Cali, a través del Fondo de Derechos Humanos; con la el Comité Permanente por la defensa de los Derechos Humanos (CPDH), y con la Asociación Nacional de Ayuda Solidaria (ANDAS). La aparición que tiene la Gobernación para con la ejecución del proyecto es una alianza de credibilidad y de validación, y en algunos casos de acompañamiento, en términos de espacios de discusión. Lo cual le da mayores y mejores garantías a la CEAR dentro del Departamento. Todas las cuestiones presupuestarias vienen de la ayuda del Ayuntamiento de Madrid y la Unión Europea.

El proyecto se implementa desde 2008 $y$ ha tenido varias fases, y uno de los resultados del mismo fue la implementación de la oficina del migrante, que empezó a funcionar en marzo del 2009. En esa oficina se brinda información gratuita a la gente sobre las vías regulares de migración a España, no se hacen trámites, no es bolsa de empleo, ni una dependencia de España,ni se ayuda a nadie a salir del país bajo la figura de asilo.

Sin embargo, según el testimonio del abogado Borja, funcionario de esta institución en España y quien trabajó en los centros de información en el aeropuerto de Barajas, el flujo de personas registrado por la CEAR, que llega de Colombia a España, no se asemeja al número de las que toma el servicio de información en la oficina de Cali.Se esperaba,desde la CEAR, tener un flujo parecido, sin embargo, los expedientes de citas muestran que solo existen 139 casos de marzo a agosto 31 de 2009 , lo cual no cumple con las expectativas de CEAR, según testimonio de la Trabajadora Social,de la oficina de la CEAR.
Por otro lado, estas son las actividades que se han planteado en el primer tramo del proyecto de CEAR - Valle del Cauca: (1) programa de intercambios de profesionales (2) programa de formación con entidades públicas y privadas, adaptación de documentación técnica (3) centralización de la información sobre migración (4) programa de formación a migrantes potenciales (5) campaña de sensibilización (6) folletos informativos (7) medios publicitarios y (8) investigación.

Su proyecto, hasta el momento, no ha cumplido con todas las expectativas, sin embargo, es claro que el trabajo realizado hasta el momento, ha permitido una mayor claridad de los procesos para aquellos que toman la decisión de informarse. De igual forma, ha sido una base para la consolidación de la información que se tiene sobre la migración, pues motivó la creación de espacios que permitan el fortalecimiento del trato al migrante en el Departamento. Además de ser una propuesta que provoca un efecto multiplicador dentro de la sociedad.

De la misma forma, se encontró sobre, América, España, Solidaridad y Cooperación - AESCO, una organización no gubernamental de desarrollo sin ánimo de lucro, que tiene diferentes sedes internacionales específicamente en España, Ecuador y Colombia. En Colombia se encuentra en Pereira, Bogotá y Cali, donde presta servicios de información, orientación, capacitación y asesoría a las diferentes personas que piensan salir del país o regresar a él.Continuamente se encuentran investigando diferentes problemáticas en temas económicos, culturales y jurídicos relacionados con el tema migratorio. Por otro lado,formulan y gestionan diferentes 
proyectos que ayudan a mejorar la calidad de vida del migrante, ofreciéndole capacitación en diferentes opciones laborales como panadería, peluquería y técnico en administración de empresas, entre otros. Lo anterior es realizado de acuerdo con su principio de solidaridad, teniendo en cuenta el concepto de codesarrollo y los Derechos Humanos [Informe AEsCo,2008].

Actualmente, dentro de esta organización se encuentran cinco comités a través de los cuales se trabaja directamente con la comunidad: (1) Comité de información, este equipo de apoyo ayuda a la persona que piensa salir del país, informándole acerca de las condiciones sociales, económicas y políticas del país al cual va a llegar, de esta manera apoya la inserción laboral y social de acuerdo con las normatividades de cada Estado.(2) Comité de comunicaciones, este equipo de trabajo busca el apoyo conjunto con entidades gubernamentales, gremios, sector educativo entre otros, con el fin de disminuir los impactos negativos que pueden llegar a presentarse con la migración internacional. (3) Comité de investigación, este equipo de apoyo se encarga de mantener actualizadas las bases de datos con la mayor información posible de las diversas situaciones en las que se encuentran las personas antes del proceso migratorio o durante el desarrollo de éste. De esta manera obtienen diferentes variables para realizar investigaciones que les permitan cumplir con sus objetivos de ayuda al migrante. (4) Comité de trabajo social, este equipo se apoya en la información obtenida por el comité de investigación ya que de esta manera brindan asistencia psicoafectiva en los problemas ya identificados en la comunidad de estudio. Por experiencia han encontrado que brindan un papel fundamental en el proceso de "reagrupación familiar e inserción laboral”, dice Jennifer Tovar, psicóloga AESCO Cali.(5) Comité de voluntariado, en este equipo de apoyo se busca tener la participación de personas que ya han vivido la experiencia de migrar hacia el exterior y que por alguna razón han tenido que vivir diferentes problemáticas relacionadas con el tema de trata de personas, diferencias culturales, o inserción laboral entre otras [AESCO, 2008].

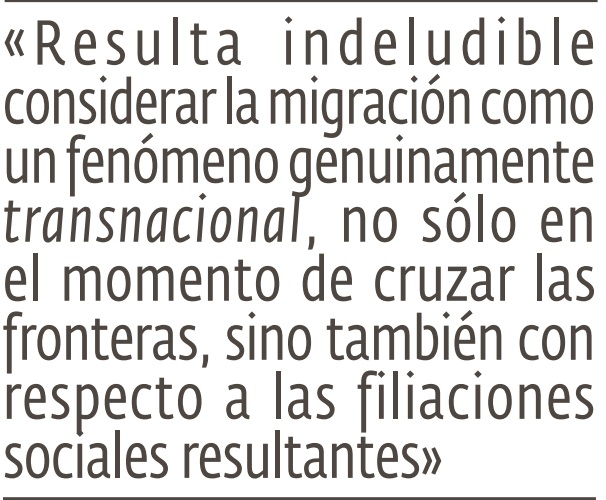

Por otro lado, esta organización ha desarrollado tres proyectos importantes de apoyo a la comunidad que tiene programado viajar, que está fuera del país o que piensa retornar. La información de estos proyectos y sus alcances fueron obtenidos a través de la entrevista directa con la señora Jennifer Tovar,psicóloga de la organización. Estos son:

Primero,la prevención de la migración desordenada, con el cual se buscaba ofrecer a las personas residentes en los municipios de Risaralda, Valle del Cauca y la ciudad de Bogotá suficiente información para prevenir problemas futuros con un 
proceso migratorio mal gestionado, que pueda llegar a atentar contra la calidad de vida del migrante, además de desarrollar estrategias educativas en la formación de artes y oficios como alternativas para mejorar el nivel de empleo en el país de llegada.

Segundo, los usos productivos a las remesas laborales, con este proyecto se buscó darle un uso productivo a todos aquellos dineros enviados al país como resultado de años (o meses) de esfuerzo y trabajo de las personas que han migrado y cuyos familiares han quedado en el país o de aquellos que piensan retornar y desarrollar una idea de negocio propio.

Tercero, el programa integral de retorno asistido, este proyecto nació a mitad del presente año (2009), cuando viene a presentarse la difícil situación financiera mundial y por la cual muchos de los migrantes perdieron su empleo,y por lo que piensan regresar al país, para lo cual el Gobierno Nacional, en conjunto con AESCO, brinda la posibilidad económica de regresar a cambio de la entrega voluntaria de la residencia española y al momento de regresar a Colombia finalizaría el proceso de ayuda financiera.

Con todo lo anteriormente realizado por AESCO, vale la pena destacar que, a pesar del apoyo que brindan al migrante o posible migrante en diferentes capacitaciones y charlas importantes en su plan de viaje o posible retorno,no brindan asesoría en la parte jurídica o legal dentro de los aeropuertos. Sin embargo, a través de contactos ayudan en la comunicación con personas encargadas de manejar estos temas con más propiedad.

Dentro de las organizaciones con las que trabajan la asesoría financiera y de inversión de remesas, han desarrollado distintos convenios entre los cuales se encuentran diferentes instituciones como Bancoldex y el Banco de la Mujer [entrevista Jennifer Tovar, psicóloga AEsco Cali]. De esta manera se le brinda, por ejemplo, a la persona retornante, un proceso de acompañamiento acerca de lo que le gustaría hacer con el dinero que logró obtener durante su estadía en otro país; se le invita a participar en un fondo, si no sabe qué hacer aún, o en microcréditos, con el fin de empezar su propia empresa con un plan de negocio de emprendimiento. Además, ofrecen programas educativos en alianza con el Servicio Nacional de Aprendizaje (SENA) sin costo alguno, la única condición es que la persona desee capacitarse. En lo que va corrido del año (2009) han sido beneficiadas 250 personas, lo que demuestra su compromiso con la comunidad.

La conformación de estas entidades ha permitido que cada vez sea más evidente la necesidad de crear espacios de atención al migrante, donde se les provea de herramientas que le garanticen mejores niveles de vida y superación de limitantes en los países de destino. Estas tres (3) entidades han llamado la atención a las entidades estatales, como el gobierno departamental y el Gobierno Nacional, poniendo en manifiesto los vacíos que ha dejado el desentendimiento por parte de ellos en años anteriores dada la descordinación.

En palabras de la socióloga Saskia Sassen "Uno dice globalización y se piensa en algo que está por fuera de todas las instituciones.Lo que sucede es que el Estado se globaliza, especialmente el Poder Eje- 
cutivo, pero la Legislatura se domestica". ${ }^{34}$ Lo que deja claro que el proceso de globalización genera una necesidad por parte de los Estados para mantener relaciones, no sólo de poder, sino de ayuda mutua en diversos temas que, como el fenómeno migratorio internacional de personas, a través de sus instituciones internacionales, que para nuestro caso de estudio en el Departamento del Valle del Cauca serían las anteriormente mencionadas, mantienen vínculos transnacionales entre los migrantes, su país de origen y aquellos a donde llegan a residir.

Sin embargo, Sassen especifica que la legislatura se domestica porque a pesar de que se da un proceso de traslado internacional de personas, es necesario mantener un orden y control a través de las diversas legislaturas estatales, lo que hace que cada país trabaje por desarrollar políticas públicas que generen más espacios para tratar temas relacionados con el tema migratorio; este proceso es el que desarrolla el Gobierno Nacional, a través del Ministerio de Relaciones Exteriores, la Gobernación Departamental y las instituciones internacionales de ayuda al migrante.La idea sería pues, pensar global, mientras se actúa localmente.

Son justamente estas instituciones internacionales las que ayudan a disminuir los riesgos de que se genere un proceso migratorio transnacional desordenado, dado que con sus programas ayudan a que el migrante y sus familias tomen conciencia de los diferentes flagelos en los que se puede convertir una mala realización de un proceso migratorio. El concepto de transnacionalismo manifiesta claramente

34 Sassen, Saskia. Beyond Sovereignty: Inmigration policie Making today, Social Justice, 23(3):9-20,1996a. las relaciones personales de doble vía que se desarrollan con los nexos sociales que se presentan cuando el migrante sale de su país de origen y cuando es recibido en el país de destino,en esta parte del proceso es donde tienen mayor participación las instituciones no gubernamentales, dado que buscan ser un vínculo de apoyo y respaldo en el ámbito social, económico, político y cultural. Es por ello que es de vital importancia su aporte al desarrollo de una política pública más incluyente dada su relación directa con la población migrante.

Por otro lado, el Valle del Cauca dentro de su desarrollo y avances en legislaturas propias ha presentado sólo un documento que pretende cobijar todas las necesidades y variables que han encontrado por las investigaciones realizadas por las instituciones nacionales e internacionales con sede en la ciudad de Santiago de Cali, la Ordenanza No.270 antes mencionada, que muestra un mayor interés por los temas referentes al tema migratorio.

Como se discutió a lo largo del texto, se encontró que el transnacionalismo es la teoría que más se relaciona con las vivencias que mantienen los colombianos en el exterior. La solidez en términos de relaciones familiares, ha permitido crear vínculos que sobrepasan las fronteras del país, originando una dependencia, no sólo en términos económicos, sino desde todas las dimensiones existentes en el campo social, afectivas, políticas y consuetudinarias.

El auge industrial disminuyó, en gran, parte los ingresos de los campesinos colombianos, quienes se vieron obligados a migrar a la ciudad en busca de oportunidades, esto conllevó a un incremento 
de la población urbana que socavó los recursos existentes en la urbe y que creó un desequilibrio, que se vio fuertemente arraigado con la aparición de los grupos armados y el narcotráfico. La violencia de los 70 provocó fuertes desajustes en la vida de los pobladores vallecaucanos siendo una de las grandes motivaciones para migrar a otros países, especialmente los desarrollados, que les permitieran tener mejores condiciones de vida y opciones de ingresos.

La historia del Valle del Cauca, su desarrollo acelerado y superior a muchos departamentos de Colombia, muestra por qué posee la mayor tasa de migración interna y externa, según el censo del DANE para el año 2005. La inminente búsqueda de alternativas para migrar - asilo político, matrimonio falso, paso ilegal, entre otras opciones- dejó entrever la gran "mafia" que hay detrás de las Migraciones Internacionales y que se beneficia de la ausencia del Estado y de actores no gubernamentales. Es así como, la falta de credibilidad en las entidades estatales y su ausencia, dio paso a la conformación de entidades no gubernamentales que velan por el pleno goce de los Derechos Humanos Internacionales por parte de los que están lejos de su país de origen. De igual manera,las entidades del tercer sector han luchado por la desaparición de la corrupción creada dada la necesidad de mejores condiciones de supervivencia y de vida que demandan la mayoría de migrantes.

La Fundación Esperanza ha sido una de las organizaciones en Colombia con más años de experiencias, la cual cuenta con un material bibliográfico muy enriquecido.Esta entidad permitió dar mayor credibilidad y fundamentos al documento
legal,Ordenanza No.270, por la que se rige la Gobernación del Valle del Cauca hasta el momento (2009) sobre el tema de la migración. De igual manera,las entidades españolas CEAR Y AESCO, vienen realizando un trabajo impulsado por entidades gubernamentales españolas, las cuales buscan la integración de los colombianos con sus familias en el país y el desarrollo de la misma a través de programas que permitan un mayor fortalecimiento de sus intereses económicos, culturales y políticos en el país de origen.

\section{Conclusiones}

La Gobernación del Valle del Cauca, con la creación de la Ordenanza No. 270 del 17 de diciembre de 2008, permitió dar el primer paso hacia la creación de mejores alternativas de seguridad e integridad de los Derechos Humanos e Internacionales de los vallecaucanos en el exterior. Sin embargo,es evidente la necesidad de crear índices de medición que permitan indagar sobre la evolución óptima de las múltiples funciones y objetivos establecidos por el Consejo Departamental de Atención Integral para la Migración.

Otra de las conclusiones principales de la investigación es sobre la evidente necesidad de iniciar actividades que garanticen el cumplimiento de lo estipulado en los documentos de trabajo del Gobierno y de entidades no gubernamentales que tratan sobre los temas de Migración Internacional. De lo contrario, se estaría dejando tan sólo por escrito las necesidades que el Gobierno Departamental observa en la población migrante y las familias que residen en el Valle del Cauca.

Con el documento CONPES 3603, queda claro que la Gobernación del Valle del Cauca no posee una política propia, 
sino que se rige por lo planteado desde el Gobierno Nacional, y que su trabajo se encuentra enfocado hacia uno de los ítems de la Política Integral Migratoria desarrollada por el Gobierno Nacional; la de desarrollar diferentes convenios con instituciones que se encuentren en la ciudad de Cali y en los cinco municipios donde se presenta, en mayor medida, el fenómeno migratorio.

En este orden de ideas, el desarrollo del documento CONPES sirvió como base para el conocimiento de mucha información que se tenía de manera empírica por las personas migrantes, pero que se necesitaba obtenerse de manera estadística [entrevista con Ximena Botero de la Torre, Directora del Programa Colombia Nos Une, Ministerio de Relaciones Exteriores] para poder enfocar las próximas decisiones del Gobierno frente al tema y saber a qué problemáticas nacionales se debe hacer frente de manera inmediata. Sin embargo, al ser un documento nacional no enfatiza en ciertos puntos que afectan directamente al Departamento del Valle del Cauca y que deben ser analizados por la Gobernación de forma más directa. Hasta el momento sólo se ha enfatizado en la creación de vínculos con instituciones que presenten información al público, pero no desde la institución misma. Es decir, el medio para relacionarse con los ciudadanos es a través de un único centro de información, ubicado en el centro de la ciudad de Santiago de Cali. Sin embargo es necesaria una mayor divulgación que ayude a controlar y minimizar el problema de Trata de Personas. Flagelos que por el desconocimiento general,o por información errada acerca del costo de las asesorías, no son prevenidos y toman decisiones riesgosas para su vida (según testimonio de un representante de CEAR).

$\mathrm{Si}$ bien es cierto que fueron muchas las recomendaciones que se obtuvieron con el CONPES ,en tema de política migratoria,y que estas a su vez necesitan de diferentes organizaciones del Gobierno Nacional como el Departamento Administrativo de Seguridad (DAS), el Ministerio de Educación Nacional, de Cultura, del Interior, el SENA, entre otros. Para poder llevarlas a cabo es necesaria la coordinación de todas las entidades involucradas en los múltiples aspectos causales del fenómeno migratorio. Ya se ha comenzado a trabajar en múltiples aspectos como el de salud, acerca de las cotizaciones con el Seguro Social y el convenio con Western Union para realizar los pagos, aunque estas instituciones son conscientes de que son muy pocas las personas afiliadas a este proceso, entienden que lleva algo de tiempo el que toda la comunidad migrante se entere de los proceso de formalización.Sin embargo, en términos generales, los actores involucrados en el proceso de implementación demuestran un positivismo frente a los resultados que se han logrado en lo corrido del año. Por otro lado, se han desarrollado convenios con el Banco de Comercio Exterior de Colombia (Bancoldex), ${ }^{35}$ con el fin de brindar apoyo a proyectos de creación de empresa para aquellas personas que regresan a Colombia y que cuentan con cierto capital, pero necesitan de más recurso para materializar sus ideas. Es muy pronto para lanzar conclusiones

$35 \mathrm{El}$ Banco de Comercio exterior (BANCOLDEX) o Banco Colombiano de Comercio Exterior, fue creado mediante el artículo 21 de la Ley ${ }^{\text {a }}$ de 1991.Es una sociedad anónima de economía mixta, de orden nacional, organizada como establecimiento de crédito bancario y vinculado al Ministerio de Comercio Exterior 
contundentes sobre el documento CONPES 3603 , sólo queda decir que su formulación es ambiciosa, necesaria y que, en el Valle del Cauca, es mucho el camino que su implementación necesita recorrer.

\section{Bibliografía}

AEsco. Portafolio de producto, Hoja No. 3, 2008.

BAUBÖск, Rainer. Hacia una teoría política del Transnacionalismo migrante, öaw, 2003,2 pág.

BETANCOURT ECHEVERRY, Darío.Mediadores, rebuscadores, traquetos y narcos, Valle del Cauca 1980-1997. Antropos.

CÁRDENAS, M. Y C. MEJÍA (2006) "Migraciones Internacionales en Colombia: ¿Qué sabemos?". Documentos de Trabajo $\mathrm{N}^{\circ} 30$, Fedesarrollo. 2005

CASTLES S. Globalization and migration: Some Pressing Contradictions, International Social Science Journal, 1998:182.

CASTLES, Stephen, Factores que hacen y deshacen las políticas migratorias, 2006.

Collins, Charles David. Formación de un sector de clase social: La Burguesía Azucarera en el Valle del Cauca durante los años treinta y cuarenta.

CONSEJO NACIONAL DE POLÍTICA ECONÓMICA Y SOCIAL - CONPES, Ministerio de relaciones Internacionales, 2009:31.

GUARNIzo, Luis Eduardo (2004), "La migración transnacional colombiana: Implicaciones teóricas y prácticas". Memorias del Seminario sobre migración Internacional colombiana y la conformación de sociedades transnacionales, Junio 18 y 19 de 2003

HELD, D., A. MCGREW, D. GOLDBLATT, and J. PERRATON.(1999). Global Transformations: Politics, Economics and Culture. Polity and Stanford University Press.
HERRERA CARASSOU, Roberto.La perspectiva teórica en el estudio de las migraciones, 2006, 227 págs.

KRUIJT Dirk, MAIGUASHA Franklin, VELLINGA Menno. Industrialización y desarrollo regional en Colombia, El desarrollo industrial en el Valle del Cauca.1982.

LONDOÑo MOTTA, Jaime Eduardo. De una región decimonónica a una región nacional: representaciones historiográficas de la creación del Departamento del Valle, Universidad Icesi, 2008.

MEYER,Eytan.Teoría de las políticas migratorias, un análisis comparado,Zona Abierta, España, 2006, 90-125pg).

MOTTA GONZALEZ, Nancy. Las dinámicas culturales y la identidad vallecaucana, Ponencia presentada en la celebración de los 95 años del Departamento del Valle del Cauca, patrocinada,2007.Ordenanza $270 \mathrm{del}$ 17 de Diciembre del 2008.

PORTES, A. Convergencias teóricas y evidencias empíricas en el estudio del Transnacionalismo de los inmigrantes. Migración y Desarrollo, 2005, primer semestre, p.2-19.

SASSEN, Saskia. Una sociología de la globalización, Katz Editores, Argentina, 2007.

SASSEN, Saskia. Beyond Sovereignty: Inmigration policie Making today,Social Justice, 23(3):9-20,1996a.

Tomado de http://www.hegoa.ehu.es/dossie$\mathrm{rra} /$ migracion/Portes-2003=transnacionalevidencia.pdf

Tomado de la oIM, www.oim.org

\section{Documentos jurídicos}

Consejo Nacional de política Económica y social, Conpes 3603, Ministerio de Relaciones Exteriores

Documento Ordenanza No. 270 del 17 de diciembre de 2008 